Journal of Advanced Research in Fluid Mechanics and Thermal Sciences

Journal homepage: www.akademiabaru.com/arfmts.html ISSN: 2289-7879

\title{
MHD Peristaltic Transport of Bingham Blood Fluid with Heat and Mass Transfer Through a Non-Uniform Channel
}

\author{
Nabil Tawfik Eldabe ${ }^{1}$, Mohamed Abouzeid ${ }^{1}$, Hamida A Shawky, ${ }^{2}{ }^{*}$ \\ Department of Mathematics, Faculty of Education, Ain Shams University, Roxy, Heliopolis, Cairo, Egypt \\ Department Mathematics, Faculty of Science (Girls section), Al-Azhar University, Nasr City, Cairo, Egypt
}

ARTICLE INFO ABSTRACT

\section{Article history:}

Received 8 May 2020

Received in revised form 15 August 2020

Accepted 19 August 2020

Available online 13 November 2020

\section{Keywords:}

Non-Newtonian Bingham fluid; magnetohydrodynamic flow; peristaltic flow; heat and mass transfer

\begin{abstract}
In the present work, the flow of non-Newtonian Bingham blood fluid through nonuniform channel is investigated. The fluid is electrically conducting, and the external uniform magnetic field is applied on this motion. The heat and mass transfer are taken in consideration, so, Soret and Dufour effects are studied. The problem is modulated mathematically by a system of non-linear partial differential equations which govern the velocity, temperature and concentration distributions. The system of these equations is simplified under the assumptions of long wavelength and low Reynolds number, then it is solved analytically by using homotopy perturbation technique. These distributions are obtained as a function of the physical parameters of the problem. The effects of these parameters on the obtained solutions are discussed numerically and illustrated graphically through a set of figures. These parameters play an important role to control the values of solutions. The used Bingham model is applicable for the physiological transportation of blood in arteries.
\end{abstract}

Copyright $@ 2021$ PENERBIT AKADEMIA BARU - All rights reserved

\section{Introduction}

Non-Newtonian blood flow with heat and mass transfer problems have received much attention due to their applications in many engineering and physiological processes and because the traditional Newtonian fluid cannot precisely describe the characteristics of the real fluids. When heat and mass transfer occur together through the fluid motion, then the relations between the fluxes and the derived potentials are of more intricate nature, and the energy flux can be generated not only due to temperature gradients, but also, by composition gradients as well. In this case the energy flux is called Dufour or diffusion-thermo effect and the mass flux is called Soret or thermal-diffusion effect. The peristaltic motion of fluid through a channels of wave walls works on mixing and transporting the fluid in the direction of wave propagation. It is an inherent property of many tubular organs of

\footnotetext{
* Corresponding author.

E-mail address: hamidashawky2020@gmail.com
}

https://doi.org/10.37934/arfmts.77.2.145159 
human body. It plays an indispensable role in transporting many physiological fluids in the human body and occurs in various situations. The study of peristaltic transport with heat and mass transfer of non-Newtonian fluid has attracted much attention recently, because of its relevance to biological systems industrial and bio-engineering and medical applications. Some recent investigations which consider MHD peristaltic motion of Newtonian and non-Newtonian fluids with heat and mass transfer are mentioned in the studies [1-6]. Tang and Fung and Gopalan [7,8] discussed blood flow by considering the microscopic blood vessels as a channel with permeable walls. They called the blood space as channel and tissue space as the porous layer. The effect of heat transfer on peristaltic transport of Newtonian fluid through a porous medium in an asymmetric vertical channel was discussed by Vasudev et al., [9]. Also, heat and mass transfer of MHD unsteady Maxwell fluid flow through porous medium past a porous flat plate was analyzed by El-dabe et al., [10]. There are several studies of peristaltic transport with different fluids under the effect of different external forces, as mentioned in [11] and see also, [12-14].

To the best of our knowledge, no investigation has been made yet to investigate the effect of slip velocity on peristaltic transport for Bingham-plastic fluid in the presence of Hall current and heat transfer. Recently, the effect of magnetic field on viscous fluid has been reported for treatment of the following pathologies: Gastroentric pathologies, rheumatisms, constipation and hypertension that can be treated by placing one electrode either on the back or on the stomach and the other on the sole of the foot; this location will induce a better blood circulation. El-dabe et al., [15] have studied heat and mass transfer of a steady slow motion of a Rivilin-Ericksen fluid in tube of varying cross-section with suction. The effect of electroosmotic on non-Newtonian blood fluid in a wavy tube, in which the flow is induced by a wave traveling on the tube wall, is investigated by Nadeem et al., [16]. The incompressible flow with radially varying magnetic field of non-Newtonian fluid, between two co-axial tubes under the considerations of long wave length and law Reynolds number, is discussed by El-dabe and Abou-zeid [17].

On the other hand, visco-plastic fluids are characterized by the absence of deformations when the applied load is below a fixed force. Bingham fluid is a special class of viscoplatic fluids named so after Bingham [18], who described several types of paints using this definition. Viscoplastic fluids constitute a very important class of non-Newtonian fluids. The modelling of Bingham materials is of crucial importance in industrial applications, since a large variety of materials (e.g. foams, pastes, slurries, oils, ceramics, etc.) exhibit the fundamental character of viscoplasticity, that is the capability of lowing only if the stress is above some critical value [19-21]. However, Bingham model is not amenable to numerical analysis because in some complex applications, some parts of material flow behave as a solid. This causes difficulties in tracking the shape and the location of the yield surfaces and applying two different constitutive equations across them. In addition, at vanishing shear rates, the apparent viscosity in Bingham model becomes infinite, which leads to a discontinuity and numerical difficulties. To overcome these issues, Papanastasiou proposed a modified Bingham model to approximate the rheological behavior of Bingham type materials [22-24].

The main aim of this study focus on investigating the effects of heat and mass transfer on peristaltic flow of blood inside a non-uniform channel. The blood is represented by Bingham model and streams through a porous medium under the effect of external uniform magnetic field. Furthermore, thermal diffusion and diffusion thermo effects with ohmic and viscous dissipation and chemical reaction are considered. The problem is modulated mathematically by using the conservation of mass and momentum, with energy and mass equations. These equations are represented in a system of coupled non-linear partial differential equations which are simplified by using long wavelength and low Reynolds number approximations. Then, they are solved analytically by using homotopy perturbation technique. The solutions are obtained as a function of physical 
parameters of the problem. The effects of these parameters on the distributions of velocity, temperature and concentration are discussed numerically and illustrated graphically through a set of figures.

\section{Mathematical Formulations}

Consider a flow of an incompressible electrically conducting non-Newtonian fluid through a porous medium inside a non-uniform channel of flexible walls. The fluid obeys Bingham model. The system is stressed by a uniform magnetic field of strength $\underline{B_{0}}$. Cartesian coordinates $(\mathrm{X}, \mathrm{Y}, \mathrm{Z})$ are chosen as seen in Figure 1. The wall equation of non-uniform channel can be written from the symmetry about the channel axis as [5]

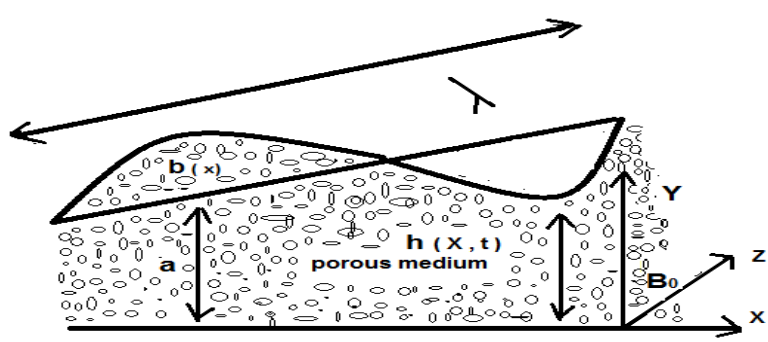

Fig. 1. Sketch of the problem

$$
h(X, t)=b(X)=b_{0}+\frac{l(X-c t)}{\lambda_{l}}+a \sin \frac{2 \pi}{\lambda_{l}}(X-c t)
$$

The constitutive equation of Bingham model which represents the relation between stress $\bar{\tau}$ and rate of strain $\bar{\gamma}$ can be written as [19]

$$
\begin{aligned}
& \overline{\bar{\tau}}=\boldsymbol{\overline { \gamma ^ { \bullet } }}, \boldsymbol{\eta}=\mu+\frac{\tau_{y}}{\left|\gamma^{\bullet}\right|}\left\{1-e^{-m\left|\gamma^{\bullet}\right|}\right\}, \\
& \overline{\overline{\gamma^{\bullet}}}=\nabla \underline{\nu}+\nabla^{T} \underline{\nu},\left|\gamma^{\bullet}\right|=\sqrt{\frac{1}{2} \overline{\overline{\gamma^{\bullet}}} \cdot \overline{\overline{\gamma^{\bullet}}}}
\end{aligned}
$$

where $m$ is the stream growth exponent which has been introduced to avoid the discontinuity in any viscoelastic model and also called a regularization parameter.

The system is stressed by an external uniform magnetic field $\underline{B}=\left(0,0, B_{0}\right) \& \underline{J}=\sigma(\underline{E}+\underline{v} \wedge \underline{B})$ and $\underline{F}=\underline{J} \wedge \underline{B}$ is the Lorenz force. We assume that the conductivity is usually small and the magnetic Reynolds number of the flow is taken to be small enough so that the induced magnetic field, external electric field and the electrical field due to the polarization of charges can be neglected. The equations governing the motion temperature and concentration can be written. 


\section{Continuity equation}

$$
\frac{\partial u}{\partial X}+\frac{\partial v}{\partial Y}=0
$$

\section{Momentum equation}

$$
\begin{aligned}
& \rho\left\{\frac{\partial u}{\partial t}+u \frac{\partial u}{\partial X}+v \frac{\partial u}{\partial Y}\right\}=-\frac{\partial p}{\partial X}+\frac{\partial \tau_{x x}}{\partial X}+\frac{\partial \tau_{x y}}{\partial Y}-\sigma B_{0}^{2} u-\frac{\mu}{k_{0}} u, \\
& \rho\left\{\frac{\partial v}{\partial t}+u \frac{\partial v}{\partial X}+v \frac{\partial v}{\partial Y}\right\}=-\frac{\partial p}{\partial Y}+\frac{\partial \tau_{y x}}{\partial X}+\frac{\partial \tau_{y y}}{\partial Y}-\sigma B_{0}^{2} v-\frac{\mu}{k_{0}} v
\end{aligned}
$$

Heat equation

$$
\begin{aligned}
& \frac{\partial T}{\partial t}+u \frac{\partial T}{\partial X}+v \frac{\partial T}{\partial Y}=\alpha\left(\frac{\partial^{2} T}{\partial X^{2}}+\frac{\partial^{2} T}{\partial Y^{2}}\right)+\frac{1}{\rho c_{p}}\left\{\tau_{x x} \frac{\partial u}{\partial X}+\tau_{x y}\left(\frac{\partial u}{\partial Y}+\frac{\partial v}{\partial X}\right)+\tau_{y y} \frac{\partial v}{\partial Y}\right\} \\
& +\frac{\sigma B_{0}^{4}}{\rho c_{p}}\left(u^{2}+v^{2}\right)+\frac{D_{T} k_{T}}{c_{s} c_{p}}\left(\frac{\partial^{2} C}{\partial X^{2}}+\frac{\partial^{2} C}{\partial Y^{2}}\right)
\end{aligned}
$$

\section{Concentration equation}

$\frac{\partial C}{\partial t}+u \frac{\partial C}{\partial X}+v \frac{\partial C}{\partial Y}=D_{T}\left(\frac{\partial^{2} C}{\partial X^{2}}+\frac{\partial^{2} C}{\partial Y^{2}}\right)+\frac{D_{T}}{T_{m}}\left(\frac{\partial^{2} T}{\partial X^{2}}+\frac{\partial^{2} T}{\partial Y^{2}}\right)-k_{c}\left(C-C_{0}\right)$

\section{Consider the following transformation}

$$
\bar{x}=X-c t, \bar{y}=Y, \bar{u}=u-c, \bar{v}=v, P(\bar{x}, \bar{y})=P(X, Y, t)
$$

where $\bar{u}$ and $\bar{v}$ are the velocity components in the moving frame $\bar{x}$ and $\bar{y}$. Then the system of equations Eq. (3)-(7) after drop par mark can be written as

$$
\begin{aligned}
& \frac{\partial u}{\partial x}+\frac{\partial v}{\partial y}=0 \\
& \rho\left\{u \frac{\partial u}{\partial x}+v \frac{\partial u}{\partial y}\right\}=-\frac{\partial p}{\partial x}+\frac{\partial \tau_{x x}}{\partial x}+\frac{\partial \tau_{x y}}{\partial y}-\left(\sigma B_{0}^{2}+\frac{\mu}{k_{0}}\right)(u+c) \\
& \rho\left\{u \frac{\partial v}{\partial x}+v \frac{\partial v}{\partial y}\right\}=-\frac{\partial p}{\partial y}+\frac{\partial \tau_{y x}}{\partial x}+\frac{\partial \tau_{y y}}{\partial y}-\left(\sigma B_{0}^{2}+\frac{\mu}{k_{0}}\right) v
\end{aligned}
$$




$$
\begin{aligned}
& u \frac{\partial T}{\partial x}+v \frac{\partial T}{\partial y}=\alpha\left(\frac{\partial^{2} T}{\partial x^{2}}+\frac{\partial^{2} T}{\partial y^{2}}\right)+\frac{1}{\rho c_{p}}\left\{\tau_{x x} \frac{\partial u}{\partial x}+\tau_{x y}\left(\frac{\partial u}{\partial y}+\frac{\partial v}{\partial x}\right)\right\} \\
& +\tau_{y y} \frac{\partial v}{\partial y}+\frac{\sigma B_{0}^{4}}{\rho c_{p}}\left((u+c)^{2}+v^{2}\right)+\frac{D_{T} k_{T}}{c_{s} c_{p}}\left(\frac{\partial^{2} C}{\partial x^{2}}+\frac{\partial^{2} C}{\partial y^{2}}\right) \\
& u \frac{\partial C}{\partial x}+v \frac{\partial C}{\partial y}=D_{T}\left(\frac{\partial^{2} C}{\partial x^{2}}+\frac{\partial^{2} C}{\partial y^{2}}\right)+\frac{D_{T}}{T_{m}}\left(\frac{\partial^{2} T}{\partial x^{2}}+\frac{\partial^{2} T}{\partial y^{2}}\right)-k_{c}\left(C-C_{0}\right)
\end{aligned}
$$

where $h(x)=b_{0}+\frac{l}{\lambda_{l}} x+a \sin \frac{2 \pi}{\lambda_{l}} x$,

$$
\begin{aligned}
& \tau_{x x}=\left\{\mu+\tau_{y}\left(m-\frac{m^{2}}{2} \sqrt{2\left[\left(\frac{\partial u}{\partial x}\right)^{2}+\left(\frac{\partial v}{\partial y}\right)^{2}\right]+\left(\frac{\partial v}{\partial x}+\frac{\partial u}{\partial y}\right)^{2}}\right)\right\} 2 \frac{\partial u}{\partial x}, \\
& \tau_{x y}=\tau_{y x}=\left\{\mu+\tau_{y}\left(m-\frac{m^{2}}{2} \sqrt{2\left[\left(\frac{\partial u}{\partial x}\right)^{2}+\left(\frac{\partial v}{\partial y}\right)^{2}\right]+\left(\frac{\partial v}{\partial x}+\frac{\partial u}{\partial y}\right)^{2}}\right]\right\}\left(\frac{\partial v}{\partial x}+\frac{\partial u}{\partial y}\right) \\
& \tau_{y y}=\left\{\mu+\tau_{y}\left(m-\frac{m^{2}}{2} \sqrt{2\left[\left(\frac{\partial u}{\partial x}\right)^{2}+\left(\frac{\partial v}{\partial y}\right)^{2}\right]+\left(\frac{\partial v}{\partial x}+\frac{\partial u}{\partial y}\right)^{2}}\right)\right\} 2 \frac{\partial v}{\partial y}
\end{aligned}
$$

Introduce the following dimensionless quantities, where the stream function $\psi$ is defined as

$$
\begin{gathered}
\left.\begin{array}{c}
u=\frac{\partial \psi}{\partial y}, v=-\frac{\partial \psi}{\partial x} \\
x^{*}=\frac{x}{\lambda_{l}}, y^{*}=\frac{y}{d}, u^{*}=\frac{u}{c}, v^{*}=\frac{v}{\delta c}, \delta=\frac{d}{\lambda_{l}}, p^{*}=\frac{d^{2}}{\lambda_{l} c \mu} p, \\
t^{*}=\frac{c}{\lambda_{l}} t, h^{*}=\frac{h}{b_{0}}, \psi^{*}=\frac{\psi}{c d}, R_{e}=\frac{c d}{\gamma}, \theta=\frac{T-T_{0}}{T_{1}-T_{0}}, \varphi=\frac{C-C_{0}}{C_{1}-C_{0}}
\end{array}\right\}
\end{gathered}
$$

Substitute from (16), the system of equations Eq. (9)-(12) after drop star mark and using the approximation of long wave length and small Reynolds number, becomes

$$
\begin{aligned}
& \frac{\partial p}{\partial x}=\left(1+\lambda_{0}\right) \psi_{y y y}-\lambda_{1} \psi_{y y} \psi_{y y y}-\left(M+\frac{1}{k}\right)\left(\psi_{y}+1\right), \\
& \frac{\partial p}{\partial y}=0
\end{aligned}
$$




$$
\begin{aligned}
& \theta_{y y}+p_{r} E_{c}\left(1+\lambda_{0}-\frac{1}{2} \lambda_{1} \psi_{y y}\right) \psi_{y y}^{2}+p_{r} E_{c} M^{2}\left(\psi_{y}+1\right)^{2}+D_{f} p_{r} \varphi_{y y}=0, \\
& \varphi_{y y}+S_{c} S_{r} \theta_{y y}-\lambda \varphi=0
\end{aligned}
$$

where

$$
\begin{aligned}
& h=1+S_{0} x+S_{1} \sin 2 \pi x \quad, \tau_{x y}=\left(1+\lambda_{0}-\frac{1}{2} \lambda_{1} \psi_{y y}\right) \psi_{y y} \quad, \lambda_{0}=\frac{\tau_{y} m}{\mu}, \lambda_{1}=\frac{\tau_{y} m^{2} c}{\mu d} \\
& M=\frac{\sigma B_{0}^{2} d^{2}}{\mu}, K=\frac{k_{0}}{d^{2}} \quad, D_{f}=\frac{D_{T} k_{T}\left(C_{1}-C_{0}\right)}{\gamma c_{S} c_{p}\left(T_{1}-T_{0}\right)}, \quad E_{C}=\frac{c^{2}}{c_{p}\left(T_{1}-T_{0}\right)}, \quad p_{r}=\frac{\gamma}{\alpha} \\
& S_{0}=\frac{l}{b_{0}}, S_{1}=\frac{a}{b_{0}}, \gamma=\frac{\mu}{\rho}, \lambda=\frac{k_{c} d^{2}}{D_{\beta}}, S_{c}=\frac{\gamma}{D_{\beta}}, S_{r}=\frac{D_{T} k_{T}\left(T_{1}-T_{0}\right)}{\gamma T_{m}\left(C_{1}-C_{0}\right)}
\end{aligned}
$$

The appropriate boundary conditions subjected to this problem in dimensionless form can be written as

$$
\left.\begin{array}{l}
\text { at } y=0 \Rightarrow \psi=0, \quad \psi_{y y}=\frac{2\left(1+\lambda_{0}\right)}{\lambda_{1}}, \theta_{y}, \varphi_{y}=0 \\
\text { at } y=h \Rightarrow \psi_{y}=0 \quad, \theta=1, \varphi=1
\end{array}\right\}
$$

\section{Method of Solutions}

The system of nonlinear ordinary differential equations Eq. (17), (19) and (20) is solved analytically by the help of homotopy perturbation technique, the method appoints a homotopy modify to obtain an approximate series solution of differential equations. In view of the HPM [2527], Eq. (7), (8) and (9) satisfy the following relations

$$
\begin{aligned}
H(p, \psi)= & L_{1}(\psi)-L_{1}\left(\psi_{10}\right)+p L_{1}\left(\psi_{10}\right)+\frac{p}{\left(1+\lambda_{0}\right)}\left[\frac{\partial p}{\partial x}+\lambda_{1} \psi_{y y} \psi_{y y y}+\left(M+\frac{1}{k}\right)\left(\psi_{y}+1\right)\right] \\
H(p, \theta)= & L_{2}(\theta)-L_{2}\left(\theta_{10}\right)+p L_{2}\left(\theta_{10}\right)+p\left(-p_{r} E_{c}\left(1+\lambda_{0}-\frac{1}{2} \lambda_{1} \psi_{y y}\right) \psi_{y y}^{2}\right. \\
& \left.-p_{r} E_{c} M^{2}\left(\psi_{y}+1\right)^{2}-D_{f} p_{r} \varphi_{y y}\right) \\
H(p, \varphi)= & L_{2}(\varphi)-L_{2}\left(\varphi_{10}\right)+p L_{2}\left(\varphi_{10}\right)+p\left(-S_{c} S_{r} \theta_{y y}+\lambda \varphi\right)
\end{aligned}
$$

With $L_{1}=\frac{d^{3}}{d y^{3}}, L_{2}=\frac{d^{2}}{d y^{2}}$ as the linear operator. The initial approximations $\psi_{10}, \theta_{10}, \varphi_{10}$ can be defined

$$
\psi_{10}=\frac{1}{\lambda_{2}}\left(1+\lambda_{1}\right)\left(y^{2}-2 h y\right)
$$


$\theta_{10}=\varphi_{10}=1$.

The stream, temperature and concentration distributions are obtained as a functions of the physical parameters of the problem as follows

$$
\begin{aligned}
& \psi=\frac{(M+1 / K)^{2}}{360 \lambda_{1}\left(1+\lambda_{0}\right)} y^{6}+\frac{M+1 / K}{120\left(1+\lambda_{0}\right)}\left(\frac{\partial p}{\partial x}+(M+1 / K)\left(\frac{1}{\left(1+\lambda_{0}\right)}-\frac{2 h}{\lambda_{1}}\right)\right) y^{5}+ \\
& \frac{(M+1 / K)}{4 \lambda_{1}} y^{4}+\frac{1}{3}\left(\frac{\partial p}{\partial x}+(M+1 / K)\left(\frac{1}{\left(1+\lambda_{0}\right)}-\frac{2 h}{\lambda_{1}}\right)\right)-\frac{(M+1 / K)^{2}}{18 \lambda_{1}\left(1+\lambda_{0}\right)} h^{3} \\
& -\frac{M+1 / K}{12\left(1+\lambda_{0}\right)}\left(\frac{\partial p}{\partial x}+(M+1 / K)\left(\frac{1}{\left(1+\lambda_{0}\right)}-\frac{2 h}{\lambda_{1}}\right)\right) h^{2} y^{3}+\frac{1+\lambda_{0}}{\lambda_{1}} y^{2}- \\
& \left(\frac{2\left(1+\lambda_{0}\right) h}{\lambda_{1}}+\frac{h^{2}}{6}\left(\frac{\partial p}{\partial x}+(M+1 / K)\left(\frac{1}{\left(1+\lambda_{0}\right)}-\frac{4 h}{\lambda_{1}}\right)\right)\right) y \\
& \theta=-\frac{4 P_{r} E_{C} M^{2}\left(1+\lambda_{0}\right)(M+1 / K)}{90 \lambda_{1}^{2}} y^{6}-\frac{P_{r} E_{C} M^{2}\left(1+\lambda_{0}\right)}{5 \lambda_{1}} \times \\
& {\left[\frac{1}{2}-\frac{(M+1 / K)}{3 \lambda_{1}}\left(h+\frac{\lambda_{1}}{2\left(1+\lambda_{0}\right)}\right)\right] y^{5}+\frac{2 P_{r} E_{C}\left(1+\lambda_{0}\right)^{2}\left((M+1 / K)+2 M^{2}\right)^{2}}{6 \lambda_{1}^{2}} y^{4}} \\
& +\left(\frac{P_{r} E_{C}\left(1+\lambda_{0}\right)^{2}}{3 \lambda_{1}}\left(\frac{\partial p}{\partial x}+(M+1 / K)\left(\frac{1}{\left(1+\lambda_{0}\right)}-\frac{2 h}{\lambda_{1}}\right)\right)+\right. \\
& \frac{4 P_{r} E_{C} M^{2} a_{1} h^{2}\left(1+\lambda_{0}\right)(M+1 / K)}{\lambda_{1}} \times \\
& \left.\left(\frac{\partial p}{\partial x}+(M+1 / K)\left(\frac{1}{\left(1+\lambda_{0}\right)}-\frac{2 h}{\lambda_{1}}\right)+\frac{2(M+1 / K) h}{3 \lambda_{1}}\right)\right) y^{3} \\
& -\frac{P_{r} E_{C} M^{2}}{2}\left(\frac{4\left(1+\lambda_{0}\right)^{2} h}{\lambda_{1}^{2}}\left(h-\frac{\lambda_{1}}{\left(1+\lambda_{0}\right)}\right)+1\right) y^{2}+\left(1+\frac{P_{r} E_{C} M^{2}\left(1+\lambda_{0}\right) h^{5}}{20 \lambda_{1}}\right) \times \\
& \left(2 h+-\frac{\lambda_{1}}{2\left(1+\lambda_{0}\right)}-\frac{(M+1 / K)}{3 \lambda_{1}}\left(h+\frac{\lambda_{1}}{2\left(1+\lambda_{0}\right)}\right)+\frac{8(M+1 / K) h}{9 \lambda_{1}^{2}}\right)
\end{aligned}
$$




$$
\begin{aligned}
& \phi=\left(S_{c} S_{r} \frac{4 P_{r} E_{C}\left(1+\lambda_{0}\right)^{2}}{12 \lambda_{1}^{2}} M^{2}+\frac{\lambda_{1}^{2}}{24}\right) y^{4}-\frac{2 P_{r} E_{C}\left(1+\lambda_{0}\right)^{2} M^{2} S_{c} S_{r}}{3 \lambda_{1}^{2}} \times \\
& \left(2 h-\frac{\lambda_{1}}{\left(1+\lambda_{0}\right)}\right) y^{3}+\left(\frac{S_{c} S_{r} P_{r} E_{C} M^{2}\left(\frac{4\left(1+\lambda_{0}\right)^{2} h}{\lambda_{1}^{2}}\left(h-\frac{\lambda_{1}}{\left(1+\lambda_{0}\right)}\right)+1\right)}{2}-\frac{\lambda_{1}{ }^{2} h^{2}}{4}+\frac{\lambda_{1}}{2}\right) y^{2} \\
& +\frac{S_{c} S_{r} h^{3} P_{r} E_{C}\left(1+\lambda_{0}\right)^{2} M^{2}}{12 \lambda_{1}^{2}}\left(2\left(2 h-\frac{\lambda_{1}}{\left(1+\lambda_{0}\right)}\right)-1+\frac{5}{2} \lambda_{1}{ }^{2} h\right)-\frac{\lambda h^{2}}{2}
\end{aligned}
$$

\section{Results and Discussion}

Figure 2-9 illustrate the effects of the physical parameters of the problem on the general behavior of the stream function, the velocity, temperature and concentration.

Figure 2 and 3 illustrate the change of the stream function $\psi$ versus the vertical coordinate $y$ for several values of the magnetic parameter $M$ and the material parameter, of Bingham model $\lambda_{0}$, respectively. It is seen, from Figure 2 and 3, that the stream function decreases with the increase of $M$, whereas it increases as $\lambda_{0}$ increases, respectively. Moreover, it is also noted that for each value of both $M$ and $\lambda_{0}, \psi$ is always negative, and all obtained curves don't intersect at the upper wall. The obtained result in Figure 2 due to the Lorentz force retards the flow. The effects of the other parameters are found to be similar to them; these figures are excluded here to avoid any kind of repetition.

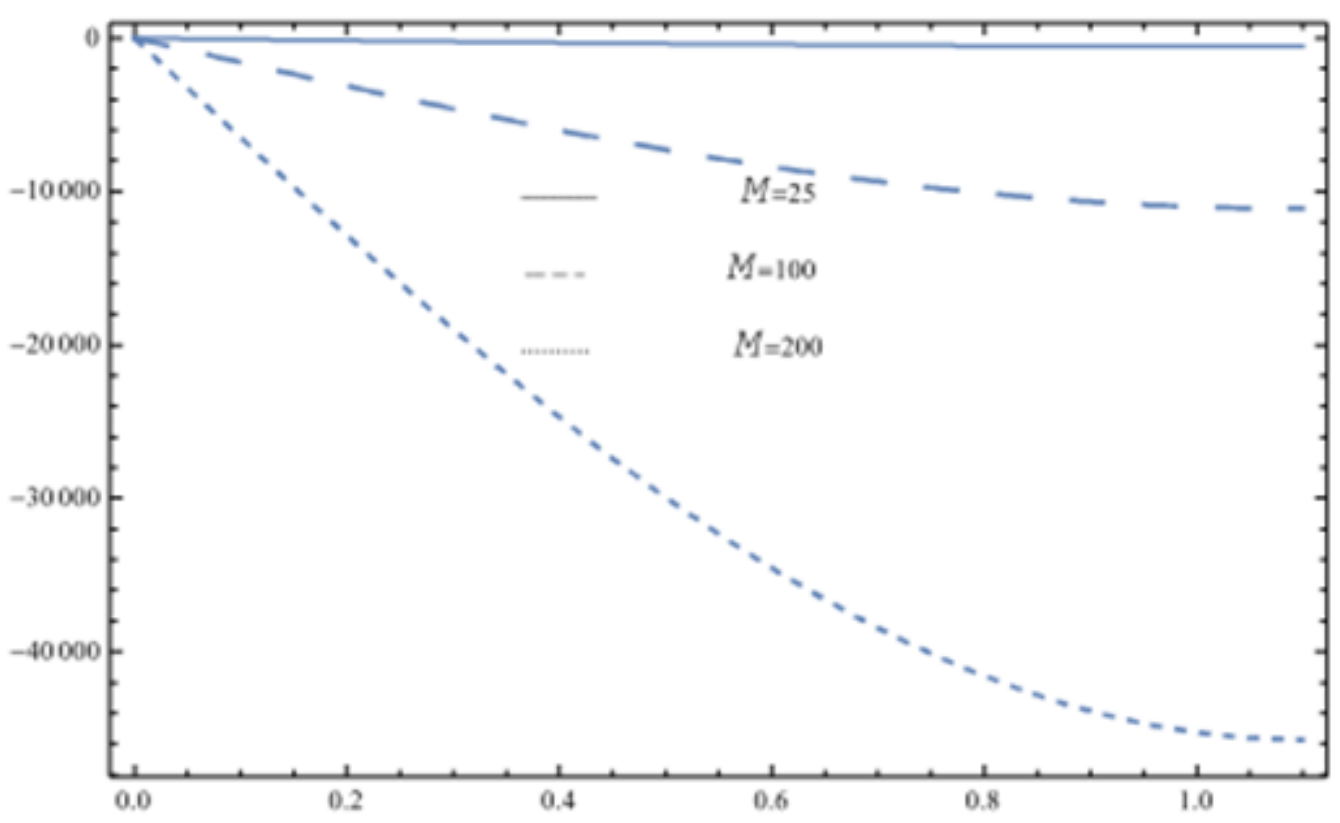

Fig. 2. The stream function $\psi$ of the fluid is drown against $y$ for different values of $E_{c}=7.5, D_{f}=2.5, K=0.2, P_{r}=1.2, \lambda_{0}=1.5, \lambda_{1}=0.1, \quad M=25,100,200$, 


$$
\begin{aligned}
& x=0.25, S_{0}=0.4, S_{1}=0.1, \frac{\partial p}{\partial x}=-10, \lambda=0.2, S_{c}=0.5, \quad S_{r}=1, \\
& h=1+S_{0} x+S_{1} \sin 2 \pi x
\end{aligned}
$$

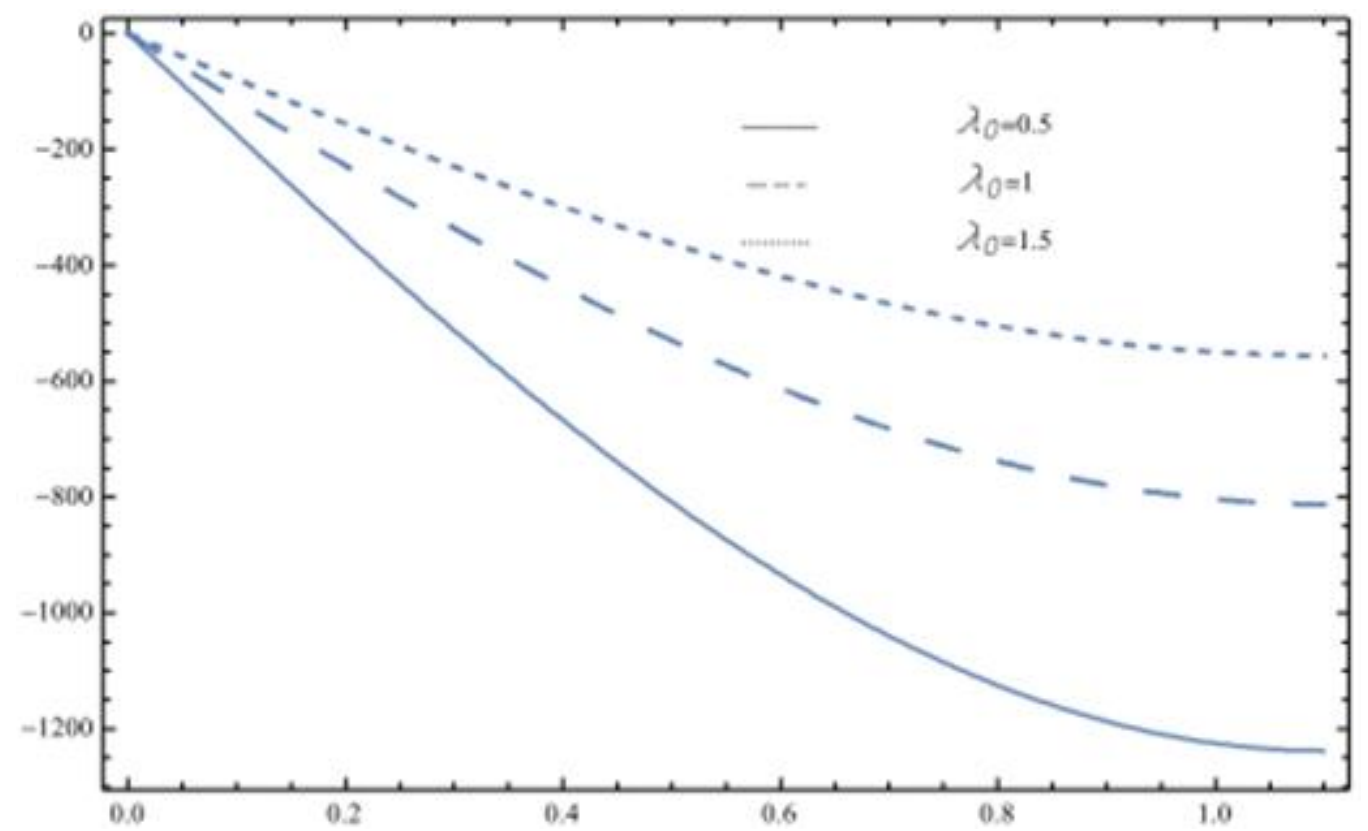

Fig. 3. The stream function $\psi$ of the fluid is drown against $y$ for different values of

$$
\begin{aligned}
& E_{c}=7.5, D_{f}=2.5, K=0.2, P_{r}=1.2, \lambda_{1}=0.1, M=25, \lambda_{0}=0,5,1,1.5, \\
& x=0.25, S_{0}=0.4, S_{1}=0.1, \quad \frac{\partial p}{\partial x}=-10, \lambda=0.2, \quad S_{c}=0.5, \quad S_{r}=1, \\
& h=1+S_{0} x+S_{1} \sin 2 \pi x
\end{aligned}
$$

The variations of the horizontal velocity $u$ with the dimensionless vertical coordinate $y$ for various values of the magnetic parameter $M$ and the material parameter of Bingham model $\lambda_{0}$ are displayed in Figure 4 and 5, respectively. The graphical results of Figure 4 and 5 indicate that the horizontal velocity decreases with increasing in the parameter $M$, while it increases by increasing the parameter $\lambda_{0}$, respectively. It is also noted from Figure 4 and 5 , that all the obtained curves don't intersect at the lower wall.

Figure 6 and 7 obtain the influence of the permeability parameter $K$ and Ecket number $E_{c}$ on the temperature distribution, respectively. It is observed that the temperature increases by increasing $K$, whereas it decreases by increasing values of $E_{c}$.

Figure 8 and 9 represent the behaviors of the concentration distribution $\varphi$ with the dimensionless vertical coordinate $y$ for different values of material parameters, of Bingham model $\lambda_{0}$ and $\lambda_{1}$, respectively. It is indicated from Figure 8 and 9 , that the concentration distribution decreases with the increase of $\lambda_{0}$, whereas it increases as $\lambda_{1}$ increases, respectively. It is also noted that the difference of the concentration distribution for different values of $\lambda_{0}$ and $\lambda_{1}$ becomes greater near the lower wall. The following explains the result in Figure 9. The material parameter of Bingham model $\lambda_{1}$ is proportional to the dynamic viscosity which is proportionally to the fluid concentration. So, $\lambda_{1}$ tends to increase the concentration. 


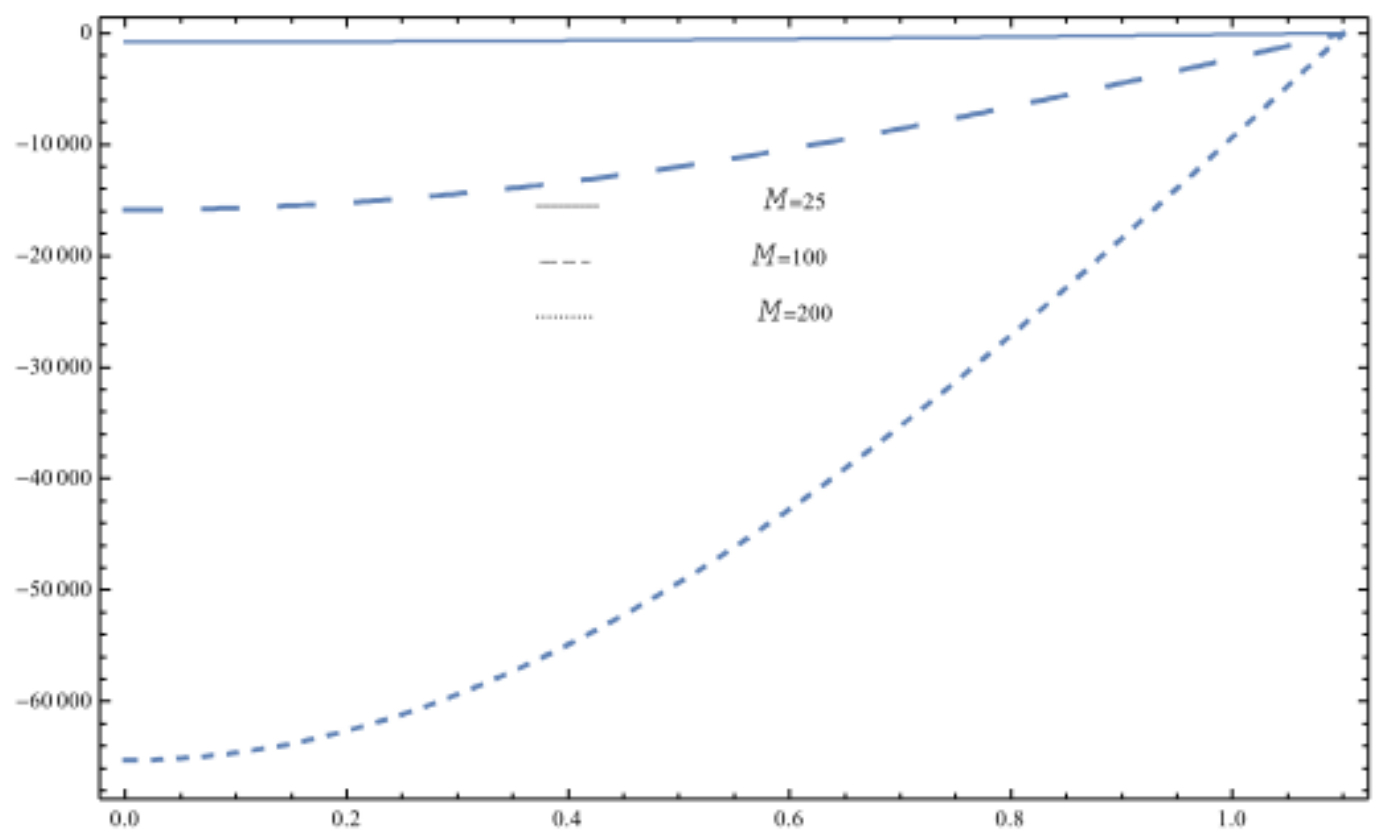

Fig. 4. The velocity function $(v)$ of the fluid is drown against $y$ for different values of $E_{c}=7.5, D_{f}=2.5, K=0.2, P_{r}=1.2, \lambda_{0}=1.5, \lambda_{1}=0.1, \quad M=25,100,200$, $x=0.25, S_{0}=0.4, S_{1}=0.1, \frac{\partial p}{\partial x}=-10, \lambda=0.2, \quad S_{c}=0.5, \quad S_{r}=1$, $h=1+S_{0} x+S_{1} \sin 2 \pi x$

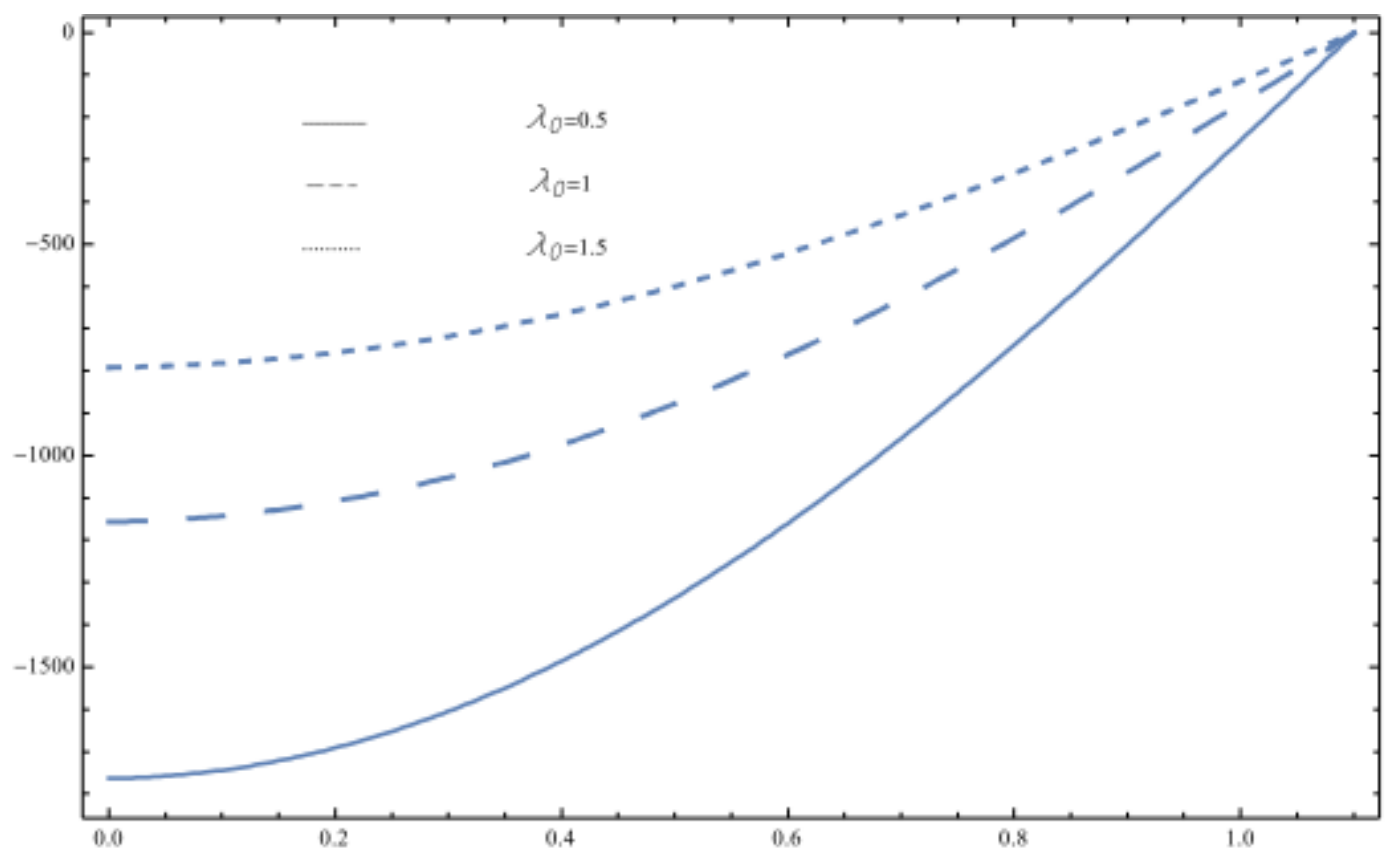

Fig. 5. The velocity function $v$ of the fluid is drown against $y$ for different values of $\lambda_{0}=0,5,1,1.5, \quad \lambda_{1}=0.1, M=25, K=0.2, \quad P_{r}=1.2, \quad E_{c}=7.5, D_{f}=2.5$, $S_{c}=0.5, S_{r}=1, \lambda=0.2, \frac{\partial p}{\partial x}=-10, S_{0}=0.4, S_{1}=0.1, \quad x=0.25$, $h=1+S_{0} x+S_{1} \sin 2 \pi x$ 


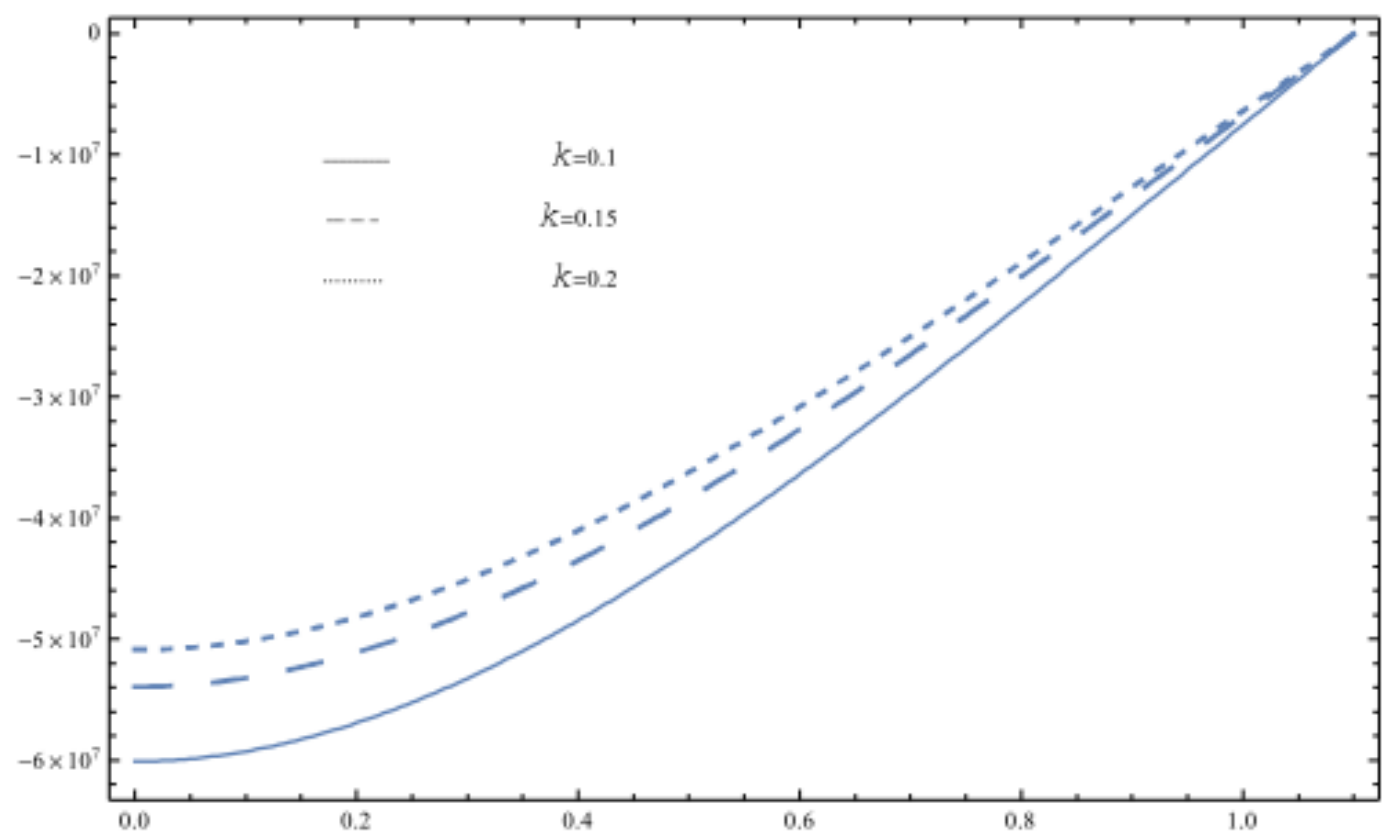

Fig. 6. The temperature function $\theta$ of the fluid is drown against $y$ for different values of $E_{c}=7.5, D_{f}=2.5, M=25, P_{r}=1.2, \lambda_{0}=1.5, \lambda_{1}=0.1, \quad K=0.1,0.15,0.2$ $x=0.25, \quad S_{0}=0.4, S_{1}=0.1, \quad \frac{\partial p}{\partial x}=-10, \lambda=0.2, \quad S_{c}=0.5, \quad S_{r}=1$, $h=1+S_{0} x+S_{1} \sin 2 \pi x$

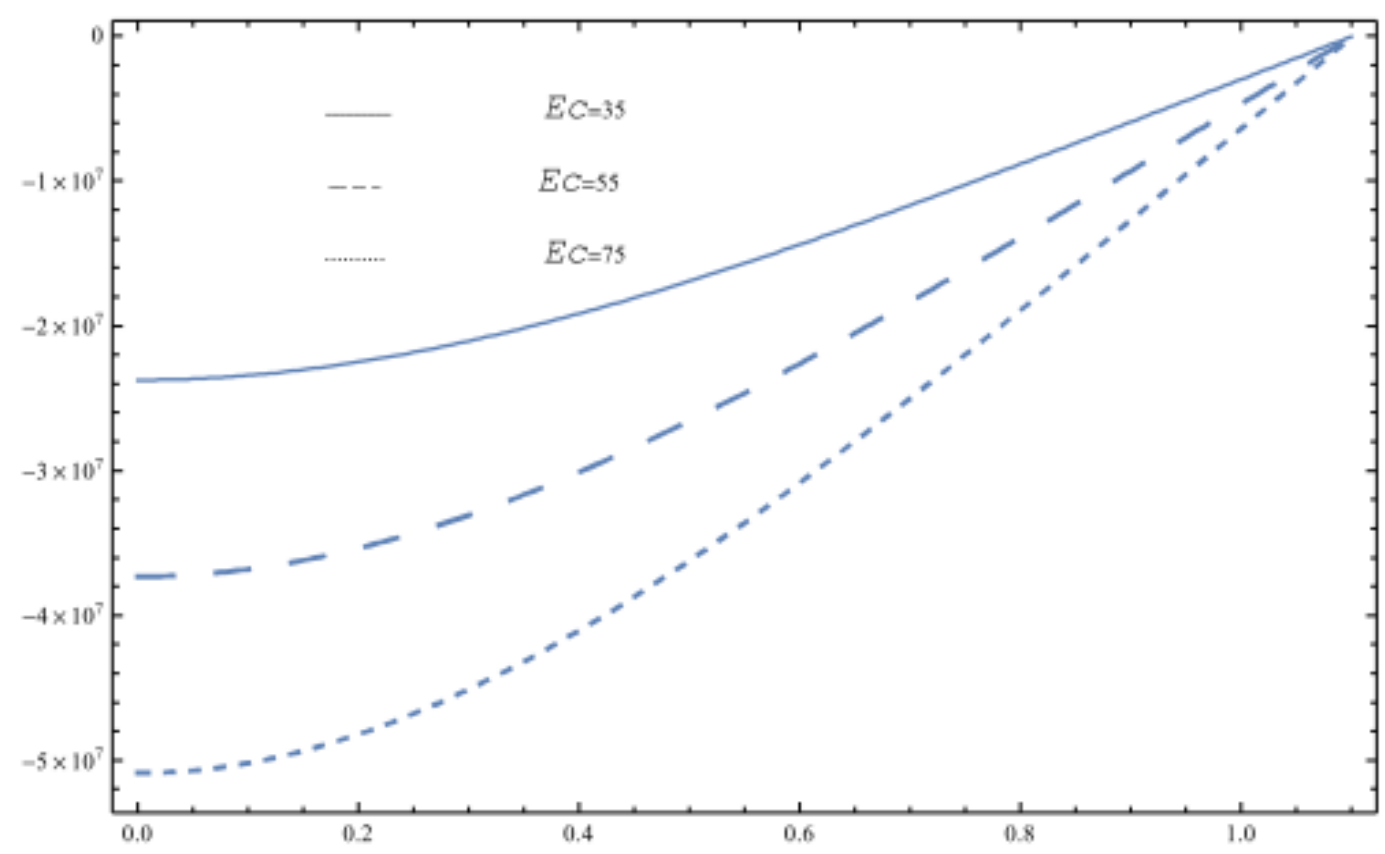

Fig. 7. The temperature function $\theta$ of the fluid is drown against $y$ for different values of $K=0.2, D_{f}=2.5, M=25, P_{r}=1.2, \lambda_{0}=1.5, \lambda_{1}=0.1, \quad E_{c}=3.5,5.5,7.5$, $x=0.1, a_{1}=0 ., \quad S_{0}=0.4, S_{1}=0.1, \quad \frac{\partial p}{\partial x}=-10, \lambda=0.2, \quad S_{c}=0.5, \quad S_{r}=1$, $h=1+S_{0} x+S_{1} \sin 2 \pi x$ 


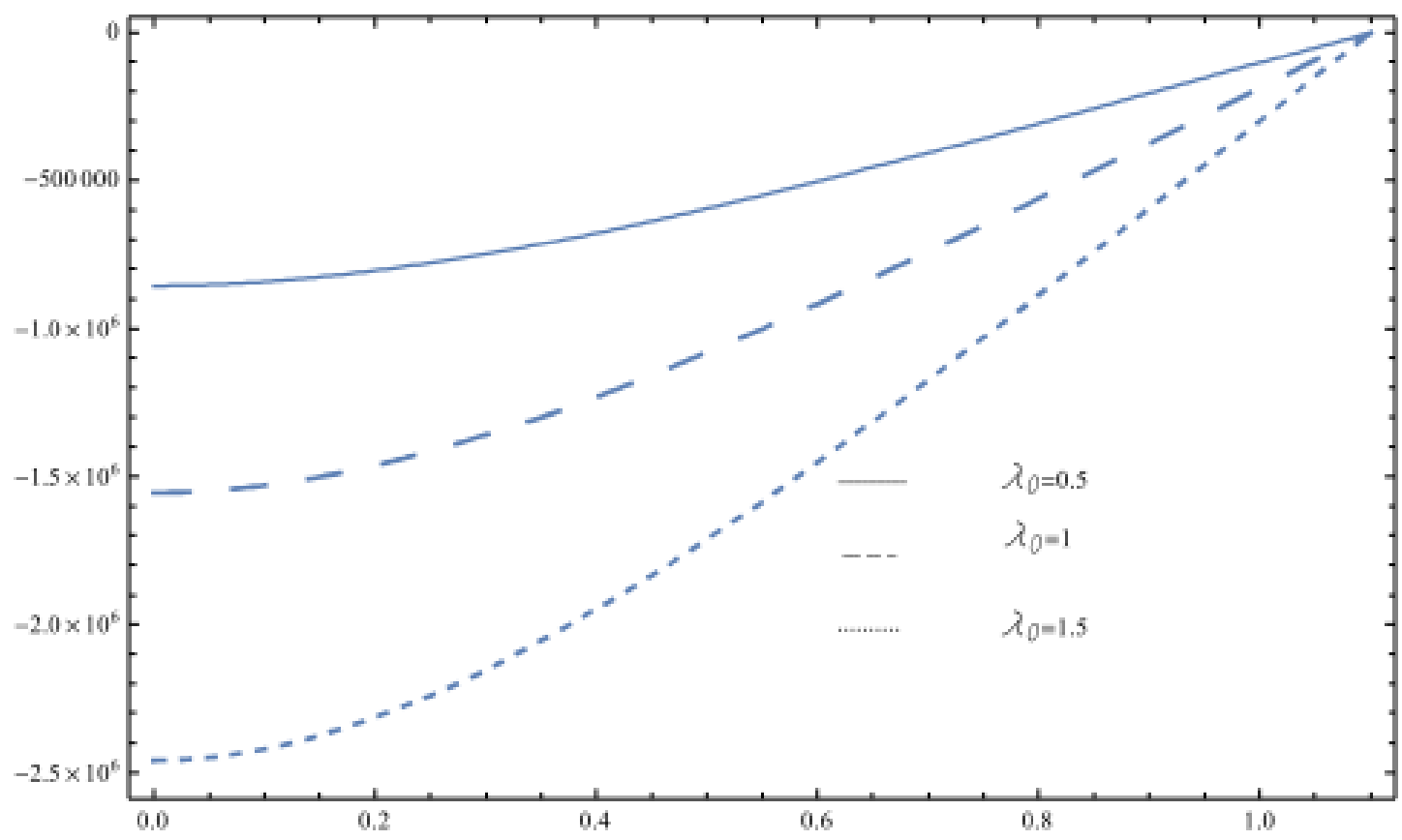

Fig. 8. The constrain function $(\varphi)$ of the fluid is drown against $y$ for different values of $S_{c}=0.5, S_{r}=1, E_{c}=7.5, D_{f}=2.5, K=0.2, P_{r}=1.2, \quad \lambda_{1}=0.1, \quad M=25, \lambda_{0}=0,5,1,1.5$, $x=0.1, a_{1}=0 ., \quad S_{0}=0.4, S_{1}=0.1, \frac{\partial p}{\partial x}=-10, \lambda=0.2$, $h=1+S_{0} x+S_{1} \sin 2 \pi x$

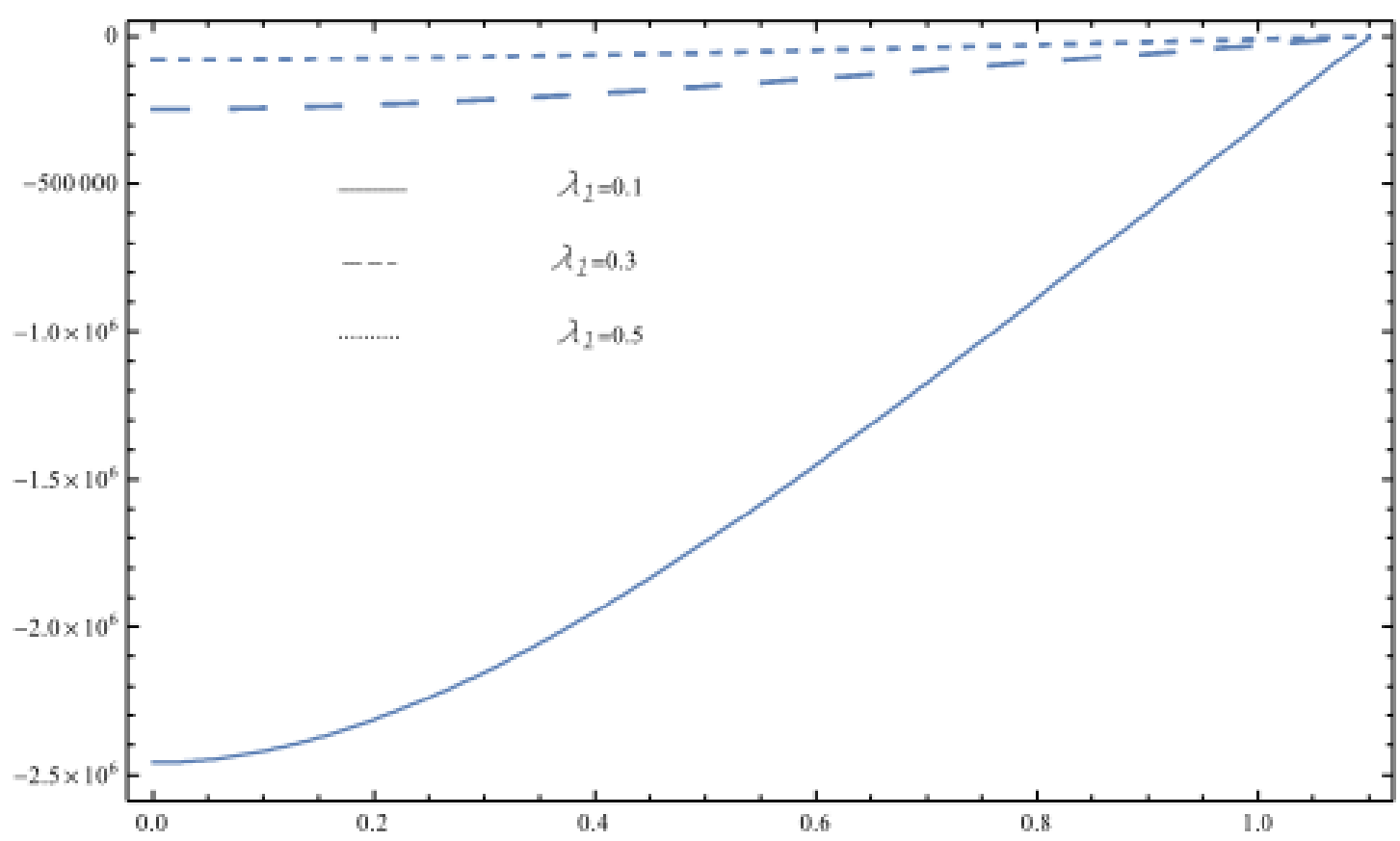

Fig. 9. The constrain function $(\varphi)$ of the fluid is drown against $y$ for different values of $E_{c}=7.5, D_{f}=2.5, K=0.2, P_{r}=1.2, \lambda_{0}=1.5, \quad M=25, \lambda_{1}=0.1,0.3,0.5$ $x=0.25, S_{0}=0.4, S_{1}=0.1, \quad \frac{\partial p}{\partial x}=-10, \lambda=0.2, \quad S_{c}=0.5, \quad S_{r}=1$, $h=1+S_{0} x+S_{1} \sin 2 \pi x$ 


\section{Conclusion}

In this study, the motion of non-Newtonian fluid with heat and mas transfer through porous media under the effect of magnetic field inside non-uniform channel is analyzed. The system of nonlinear differential equations which describe the flow is solved by using homotopy perturbation method. Moreover, the velocity, the stream function, temperature and concentration distributions are obtained as a functions of physical parameters of the problem. The effects of these parameters on these distributions are discussed numerically. The following observations have been found

i. The stream lines $\psi$ increase with the increase of each of, whereas it $\lambda_{1}$ and $\lambda_{0}, D_{f}$ decreases as $M$ increases.

ii. The horizontal velocity $u$ for different values of all parameters is always negative and becomes greater with increasing the coordinate $\eta$, but all curves don't intersect at the end of thin-layer.

iii. The temperature increases with the increase of each of $D_{f}$ and $\lambda_{1}$, while it decreases as $\lambda_{0}$, $E_{c}$ and $M$ increase.

iv. The concentration behavior is similar to the temperature behavior except that for different values of $\lambda_{1}$, the effect appears only for large values.

\section{References}

[1] Abbas, Nadeem, M. Y. Malik, and S. Nadeem. "Transportation of magnetized micropolar hybrid nanomaterial fluid flow over a Riga curface surface." Computer methods and programs in biomedicine 185 (2020): 105136. https://doi.org/10.1016/i.cmpb.2019.105136

[2] Abou-Zeid, Mohamed Y., Abeer A. Shaaban, and Muneer Y. Alnour. "Numerical treatment and global error estimation of natural convective effects on gliding motion of bacteria on a power-law nanoslime through a nonDarcy porous medium." Journal of Porous Media 18, no. 11 (2015). https://doi.org/10.1615/JPorMedia.2015012459

[3] Eldabe, N. T., G. M. Moatimid, M. Y. Abouzeid, A. A. ElShekhipy, and Naglaa F. Abdallah. "A semianalytical technique for MHD peristalsis of pseudoplastic nanofluid with temperature-dependent viscosity: Application in drug delivery system." Heat Transfer-Asian Research 49, no. 1 (2020): 424-440.

https://doi.org/10.1002/htj.21619

[4] Rashid, M., K. Ansar, and S. Nadeem. "Effects of induced magnetic field for peristaltic flow of Williamson fluid in a curved channel." Physica A: Statistical Mechanics and its Applications (2020): 123979. https://doi.org/10.1016/i.physa.2019.123979

[5] Mansour, Hesham Mohamed, and Mohamed Y. Abou-zeid. "Heat and Mass Transfer Effect on Non-Newtonian Fluid Flow in a Non-uniform Vertical Tube with Peristalsis." Journal of Advanced Research in Fluid Mechanics and Thermal Sciences 61, no. 1 (2019): 44-62.

[6] Eldabe, N. T., and M. Y. Abou-zeid. "Homotopy perturbation method for MHD pulsatile non-Newtonian nanofluid flow with heat transfer through a non-Darcy porous medium." Journal of the Egyptian Mathematical Society 25, no. 4 (2017): 375-381. https://doi.org/10.1016/i.joems.2017.05.003

[7] Tang, H. T., and Y. C. Fung. "Fluid movement in a channel with permeable walls covered by porous media: A model of lung alveolar sheet." Journal of Applied Mathematic 97, (1975): 45-50. https://doi.org/10.1115/1.3423551

[8] Gopalan, N. P. "Pulsatile blood flow in a rigid pulmonary alveolar sheet with porous walls." Bulletin of mathematical biology 43, no. 5 (1981): 563-577. https://doi.org/10.1016/S0092-8240(81)80083-3

[9] Vasudev, C., U. Rajeswara Rao, M. V. Subba Reddy, and G. Prabhakara Rao. "Effect of heat transfer on peristaltic transport of a Newtonian fluid through a porous medium in an asymmetric vertical channel." European Journal of Scientific Research 44, no. 1 (2010): 79-92.

https://doi.org/10.5251/ajsir.2010.1.3.656.666 
[10] Eldabe, Nabil T., Raafat R. Rizkalla, Mohamed Y. Abouzeid, and Vivian M. Ayad. "Thermal diffusion and diffusion thermo effects of Eyring-Powell nanofluid flow with gyrotactic microorganisms through the boundary layer." Heat Transfer-Asian Research 49, no. 1 (2020): 383-405.

https://doi.org/10.1002/htj.21617

[11] Eldabe, Nabil TM, Afaf S. Zaghrout, Hameda M. Shawky, and Amera S. Awad. "Peristaltic transport of micropolar fluid through porous medium in a symmetric channel with heat and mass transfer in the presence of generation and radiation." African Journal of Mathematics and Computer Science Research 6, no. 6 (2013): 121-129.

[12] Bhatti, M. M., and M. M. Rashidi. "Study of heat and mass transfer with Joule heating on magnetohydrodynamic (MHD) peristaltic blood flow under the influence of Hall effect." Propulsion and Power Research 6, no. 3 (2017): 177-185. https://doi.org/10.1016/i.jppr.2017.07.006

[13] Bhatti, M. M., M. Ali Abbas, and M. M. Rashidi. "Combine effects of Magnetohydrodynamics (MHD) and partial slip on peristaltic Blood flow of Ree-Eyring fluid with wall properties." Engineering science and technology, an international journal 19, no. 3 (2016): 1497-1502.

https://doi.org/10.1016/j.jestch.2016.05.004

[14] Eldabe, N. T., M. A. Elogail, S. M. Elshaboury, and Alfaisal A. Hasan. "Hall effects on the peristaltic transport of Williamson fluid through a porous medium with heat and mass transfer." Applied Mathematical Modelling 40, no. 1 (2016): 315-328. https://doi.org/10.1016/i.apm.2015.04.043

[15] El Dabe, Nabil T., Galal M. Moatimid, and Hoda SM Ali. "Rivlin-Ericksen fluid in tube of varying cross-section with mass and heat transfer." Zeitschrift für Naturforschung A 57, no. 11 (2002): 863-873. https://doi.org/10.1515/zna-2002-1105

[16] Nadeem, Sohail, Mishal Nayab Kiani, Anber Saleem, and Alibek Issakhov. "Microvascular blood flow with heat transfer in a wavy channel having electroosmotic effects." Electrophoresis 41, (2020): 1198-1205. https://doi.org/10.1002/elps.201900465

[17] Eldabe, Nabil, and Mohamed Abou-Zeid. "Radially varying magnetic field effect on peristaltic motion with heat and mass transfer of a non-Newtonian fluid between two co-axial tubes." Thermal Science 22, no. 6 Part A (2018): 24492458. https://doi.org/10.2298/TSCl160409292E

[18] Bingham, Eugene Cook. An investigation of the laws of plastic flow. No. 278. US Government Printing Office, 1917.

[19] Shahzadi, Iqra, Naveed Ahsan, S. Nadeem, and Alibek Issakhov. "Analysis of bifurcation dynamics of streamlines topologies for pseudoplastic shear thinning fluid: Biomechanics application." Physica A: Statistical Mechanics and its Applications 540 (2020): 122502.

https://doi.org/10.1016/i.physa.2019.122502

[20] Fusi, Lorenzo, and Angiolo Farina. "Flow of a Bingham fluid in a non symmetric inclined channel." Journal of NonNewtonian Fluid Mechanics 238 (2016): 24-32. https://doi.org/10.1016/i.jnnfm.2016.04.007

[21] Turan, Osman, Sahin Yigit, and Nilanjan Chakraborty. "Numerical investigation of mixed convection of Bingham fluids in cylindrical enclosures with heated rotating top wall." International Journal of Heat and Mass Transfer 108 (2017): 1850-1869. https://doi.org/10.1016/i.ijheatmasstransfer.2017.01.034

[22] Alexandrou, Andreas N., Philippe Le Menn, Georgios Georgiou, and Vladimir Entov. "Flow instabilities of HerschelBulkley fluids." Journal of non-newtonian fluid mechanics 116, no. 1 (2003): 19-32. https://doi.org/10.1016/S0377-0257(03)00113-7

[23] Mitsoulis, E. "Flows of viscoplastic materials: models and computations." Rheology Reviews (2007): $135-178$.

[24] Zhu, H., Y. D. Kim, and D. De Kee. "Non-Newtonian fluids with a yield stress." Journal of Non-Newtonian Fluid Mechanics 129, no. 3 (2005): 177-181. https://doi.org/10.1016/i.jnnfm.2005.06.001

[25] Abou-Zeid, Mohamed Y. "Implicit Homotopy Perturbation Method for MHD Non-Newtonian Nanofluid Flow with Cattaneo-Christov Heat Flux Due to Parallel Rotating Disks." Journal of Nanofluids 8, no. 8 (2019): 1648-1653. https://doi.org/10.1166/jon.2019.1717

[26] El-dabe, Nabil Tawfik, Mohamed Yahya Abou-zeid, and Ola S. Ahmed. "Motion of a Thin Film of a Fourth Grade Nanofluid with Heat Transfer Down a Vertical Cylinder: Homotopy Perturbation Method Application." Journal of Advanced Research in Fluid Mechanics and Thermal Sciences 66, no. 2 (2020): 101-113.

[27] Mohamed, Mona AA, and Mohamed Y. Abou-zeid. "MHD peristaltic flow of micropolar Casson nanofluid through a porous medium between two co-axial tubes." Journal of Porous Media 22, no. 9 (2019): 1079-1093. 
https://doi.org/10.1615/JPorMedia.2018025180 